\title{
Colpocleisis surgery in the treatment of pelvic organ prolapse in elderly women
}

\begin{abstract}
The prolapse of pelvic organs (POP) is a prevalent disease in the elderly women with a significant impact on quality of life. Symptomatic POP requires treatment, but these women most often have other medical comorbidities that limit the therapeutic possibilities. In addition, significant rates of recurrence and complications following reconstructive surgery demand simpler and faster surgeries. For this reason obliterative surgeries such as Le Fort colpocleisis returned to be appointed and new studies have demonstrated its correct indication and success rates. This article describes results of a cases series of patients undergoing colpocleisis surgery for treating of advanced pelvic organ prolapse (POP) and compare them with some literature data.
\end{abstract}

Keywords: pelvic organ prolapse, surgery, colpocleisis
Volume 4 Issue 6 - 2019

\author{
Marchesini AC,' Antunes Jr A,' Gusmão LC,'² \\ Luz LB, ${ }^{2}$ Gollop TR ${ }^{3}$ \\ 'Associate Professor of Gynecology discipline, Faculty of \\ Medicine of Jundiaí, Brazil \\ ${ }^{2}$ Graduation student in medicine, Faculty of Medicine of Jundiaí, \\ Brazil \\ ${ }^{3}$ Associate Professor of Gynecology discipline, Faculty of \\ Medicine of Jundiaí, Brazil
}

\begin{abstract}
Correspondence: Ana Carolina Marchesini, Department of Obstetrics and Gynecology, Faculty of Medicine of Jundiaí; Rua Francisco Telles\#250Vila Arens, CEP I 3202-550 Jundiaí SP-Brazil, Tel 33952100,Email anacamarchesini@gmail.com
\end{abstract}

Received: October 24, 2019 | Published: November 07, 2019

\section{Introduction}

With increasing life expectancy of the population the age-related diseases also become more frequent and require special attention. Prolapse of pelvic organs (POP) is a common condition among elderly women, with a prevalence of 25 to $40 \%$ in multiparous women., Symptomatic patient will need some treatment because the anatomical damage causes self-image distortion, affecting their quality of life, and may also cause urinary and bowel disorders. ${ }^{3}$

POP treatment may have different goals such as restoration of function and anatomy of the vagina, or improvement affected women's quality of life. For early-stage POPs, conservative treatments such as pelvic floor physiotherapy have good results, but in advanced stages surgical interventions or vaginal pessaries are required. Women are estimated to have an $11 \%$ lifetime risk of needing pelvic floor surgery and an additional $29 \%$ lifetime risk of undergoing another surgery for POP or urinary incontinence (UI) treatment. ${ }^{4}$

Even with improved quality of life reported by patients who opt for conservative treatment using pessaries, the necessary care and the difficulties of inserting and removing the same end up targeting older women to surgical treatment. Among the surgeries that can be proposed for the treatment of POP there are reconstructive and obliterative techniques. ${ }^{5}$ The first aims to restore the pelvic and perineal anatomy preserving the vaginal function. These techniques are offered to patients with better clinical conditions and who wish to maintain an active sex life, but these surgeries require more inputs, such as synthetic meshes, and show more complications intra and postoperatively. Obliterative surgeries aim only to the elimination of $\mathrm{P}_{0} \mathrm{P}_{5}$, with loss of vaginal function. They are offered to patients who no longer wish to mantain an active sex life or the use of synthetic meshes for anatomical repair; or who experience clinical complications requiring simpler intraoperative and postoperative conditions.
The Le Fort surgery is an obliterative technique that was forgotten in the surgical arsenal at the expense of vaginal hysterectomy in cases of POP. But with increasing life expectancy, the POP recurrence after hysterectomy are more frequent, requiring reoperation. Many elderly but healthy women who experience POP recurrence after hysterectomy choose obliterative surgery because of lower risk of POP recurrence. ${ }^{6}$ Recently new studies shows different experiences with Le Fort surgery; and the purpose of this article is to present the facts in a series of cases of grade 4 prolapse treated with this obliterative technique.

\section{Methods}

A retrospective observational study of a cases series was conductes in women undergoing Le Fort colpocleisis surgery between October/2016 and October / 2018, with at least 6 months of postoperative follow-up. All patients were operated at the University Hospital of Jundiaí by Pelvic Floor Surgery team of Jundiaí School of Medicine, composed of 2 senior gynecologists assisted by the team of resident medical service. The same surgical team is responsible for outpatient care before and after surgery, and assessed POP stage by POP-Q System (Pelvic Organ Prolapse Quantification) in previous gynecological examination. Measures the POP-Q are integers made in centimeters: Hymen is the point of reference $(0)$ values with + sign indicate that this structure is external to the hymen; $\mathrm{C}$ is the most distal or outward point of the cérvix, or the vaginal vault in patients hysterectomy; $\mathrm{Ba}$ is the outward or distal portion of the anterior vaginal wall; $\mathrm{Bp}$ is the outward or distal portion of the posterior vaginal wall. All patients were operated after signing surgical consent form.

All surgeries were performed with the patient in lithotomy position after spinal anesthesia. The surgical technique of Le Fort colpocleisis was as follows: vaginal mucosa was demarcated and removal flap in rectangle shape of the anterior and posterior vaginal wall, exposing the pubocervical fascia (anterior) and rectovaginal fascia (posterior); 
denuded area were sewn together front-to-back in progressive rows using 0- Vicryl interrupted suture alternating with 2.0-PDS continuous suture, until total prolapse invagination, enclosing the suture to approximate anterior and posterior vaginal mucosa in their distal portions, closing the vagina. Throughout the procedure, lateral tunnels of vaginal mucosa were maintained for eventual drainage of endometrium or cervix secretions. Finally posterior colpoperineoplasty surgery was performed to reinforce the perineum and bladder catheterization maintained for six hours.

The sample size was estimated by convenience including all patients operated in the selected period. These cases were not matched to any control group that had been treated with another type of surgical

Table I Characterization of the sample approach or with pessaries. Hospital data, preoperative evaluation and postoperative complications were extracted from medical records and arranged in Excel ${ }^{\circledR}$ database. For this initial presentation of the data, minimum and maximum values were estimated and mean and median values calculated. It was established that the minimum follow-up of patients included in the study was 6 months, and POP recurrence were considered failure cases.

\section{Results}

From October 2016 to October 2018, meeting the minimum criterion of 6 months of follow up, 19 patients were included. The mean duration of follow up was 16.3 months. The sample characteristics are presented in Table 1.

\begin{tabular}{lllll}
\hline & Minimum & Maximum & Average & median \\
\hline Age & 64 & 83 & 73.05 & 73 \\
Menopause age & 38 & 55 & 47.66 & 50 \\
Parity & 2 & 11 & 6.94 & 7 \\
Vaginal deliveries & 1 & 9 & 6.15 & 6 \\
Newborn heaviest weight (grams) & 2,900 & 5,000 & 3946 & 4,000 \\
follow up time (months) & 6 & 30 & 16.26 & 15 \\
POP-Q & & & & \\
C & +4 & 10 & +7.68 & +8 \\
Ba & +4 & +9 & +6.31 & +6 \\
Bp & +3 & +9 & +5.89 & +6 \\
\hline
\end{tabular}

The average age of patients was 73 years, all were postmenopausal and had no genital bleeding. No hysterectomies were performed in patients with preserved uterus, and 4 patients operated for vaginal vault prolapse.

Regarding POP degree, the vast majority of patients had stage 4 prolapse (15 patients) and the rest stage 3 . The measurement of these points have to be defined ranged from +4 to +10 with a median of +8 , the measurement of point $\mathrm{Ba}$ ranged from +4 to +9 with a median of +6 , and the point $\mathrm{Bp}$ ranged from +3 to +9 with a median of +6 . +6 .

In preoperative evaluation, 8 patients $(42.1 \%)$ had some type of urinary incontinence complaint - urgincontinence, stress incontinence or both. In two of these cases was performed suburethral sling during colpocleisis surgery. The other 6 patients with urinary complaints performed exclusively with the colpocleisis and posterior perineoplasty.

Only $1(5.2 \%)$ patient had an anterior vaginal wall prolapse after 8 months of follow-up. In this case the patient had already undergone hysterectomy for uterine prolapse, the preoperative assessment $\mathrm{Ba}$ $(+6)$ was greater than the $\mathrm{C}(+5)$ and posterior wall prolapse was not very pronounced $(\mathrm{Bp}+3)$. There were no serious postoperative complications such as bleeding or infection that required any surgical re-intervention or intravenous antibiotics. Three patients, after 1 week of postoperative, complained of malodorous vaginal discharge being prescribed oral antibiotic (metronidazole) with remission of symptoms.
All patients declared themselves satisfied or very satisfied with the surgery, and even the patient who presented POP recurrence did not declare to be dissatisfied because the relapse was less than the original prolapse, and she expressed desire to have further surgery.

\section{Discussion}

The POP is predominantly found in elderly women whose comorbidities are limiting factors to therapeutic choice, so POP surgery techniques have been widely studied and reissued. ${ }^{7}$ Frequent recurrences of conventional surgery, added to the complications described in meshes surgeries and management difficulties in conservative treatment with pessaries, made the vaginal obliterative surgery an interesting therapeutic option for women with no intention of sexual activity maintenance.

Pelvic floor reconstructive surgeries in older women are associated with higher rates of complications and increase up to $13.6 \%$ risk of death. ${ }^{8}$ Our sample did not show any serious adverse event, and the only complications found were vaginal infections, controlled with antibiotics without need for surgical reintervetion or suture lines damages. These findings corroborate the evidence that complications in obliterative surgery are less frequent when compared to reconstructive surgery of pelvic floor. ${ }^{8}$

There were no new cases of urinary incontinence postoperatively, and patients who were treated with transobturator sling for stress incontinence improved symptoms in our sample. Some studies report 
7.7 to $40.6 \%$ cases of occult urinary incontinence in patients who corrected advanced POP. ${ }^{9,10}$ It was not the aim of this study to evaluate bowel disorders in patients who underwent colpocleisis, but there is evidence that the correction of POP with this technique improves most symptoms of intestinal obstruction such as as fecal incontinence. ${ }^{11}$

In our sample only 1 patient had a POP recurrence (5.2\%). This patient had an asymmetric predominantly anterior POP ; and it was already a case of recurrence after uterine prolapse treated with hysterectomy. Krissi et al. showed $19.1 \%$ of recurrence in their series of 47 patients and analyzing different parameters attributed increased risk to cases with higher total vaginal length (TVL) and increased genital hiatus $(\mathrm{GH})$; possibly suggested that recurrence would be linked to greater difficulty in obliteration of the vagina. ${ }^{12}$ They observed GH and TVL mean values of 4.6 and 9.5 respectively and the recurrence group showed GH mean of 5.1 and TVL mean of 8.9. Our patient that presented recurrence had $\mathrm{GH}=4$ and $\mathrm{TVL}=7$; but the average of GH and TVL in the whole sample were 5 and 8.5, respectively.

A limitation of this study was the sample size, and this is due to the fact that until October 2016 obliterative surgeries were not performed in our hospital. But this first evaluation after two years performing colpocleisis to POP treatment encouraged us to invest in technical and reassess these data including new cases. Another limitation of this study was the evaluation of patient satisfaction after colpocleisis. We intend to apply a validated questionnaire in the next cases instead of the subjective assessment of the patient.

\section{Conclusion}

Despite the small sample size, our cases series showed similar findings with other studies reinforcing the concept that colpocleisis surgery satisfactorily solves advanced POP (stage 3 and 4) and has a low recurrence rate. For this reason it should be considered as a treatment option for women who are not interested in maintaining sexual activity, do not want the underwent meshes surgeries and want a surgical treatment with low risk of complications.

\section{Acknowledgments}

None.

\section{Conflicts of interest}

The authors declare to have no conflicts of interest.

\section{References}

1. Linder BJ, JB Gebhart, Occhino JA. Total colpocleisis: technical considerations. Urogynecol J Int. 2016;27(11):1767-1769.

2. Wu JM, Matthews CA, Conover MM, et al. Lifetime risk of stress urinary incontinence and pelvic organ prolapse surgery. Obstet Gynecol. 2014;123:1201-1206.

3. Tarver T. Older Americans 2012: Key indicators of wellbeing. J Consum Internet Health. 2013;17:114-115.

4. Lee U, S Raz. Emerging concepts pelvic organ prolapse surgery is: heal what is? Current urology reports, Los Angeles. 2011;12:62-67.

5. Park JY, Han SJ, Kim JH, et al. Le Fort partial colpocleisis in the effective treatment option for advanced apical prolapse inelderly women. Taiwanese Journal of Obstet \& Gynecol. 2019;58(2):206-211.

6. Abbasy S, K Kenton. obliterative Procedures for pelvic organ prolapse. Clin Obstet Gynecol. 2010;53:86-98.

7. S Krishnan. Geriatric pelvic organ prolapse surgery: going the extra mile. J Obstet Gynaecol India. 2017;67(6):428-431.

8. VW Sung, S Weitzen, ER Sokol, et al. Effect of patient age on Increasing morbidity and mortality following urogynecologic surgery. Am J Obstet Gynecol. 2006;194(5):1411-1407.

9. Silay K, S Akinci, Ulas A, et al. Occult urinary incontinence in elderly women and its association with geriatric condition. Eur Rev Med Pharmacol Sci. 2016;20(3):447-451.

10. Jundt $\mathrm{K}$, Wagner $\mathrm{S}$, von Bodungen $\mathrm{V}$, et al. Occult incontinence in women with pelvic organ prolapse - Does it matter? Eur J Med Res. 2010;15(3):112-116.

11. Gutman RE, CS Bradley, W Ye, et al. Pelvic floor disorders Network. Effects of colpocleisis on bowel symptoms among women with severe pelvic organ prolapse. Urogynecol Int J. 2010;21(4):461-466.

12. Krissi H, Aviram A, Eitan R, et al. Risk factors for recurrence after Le Fort colpocleisis for severe pelvic organ prolapse in elderly women. Int J Surg. 2015;20:75-79 